\title{
BASIC POLYNOMIAL INEQUALITIES ON INTERVALS AND CIRCULAR ARCS
}

\author{
TAMÁS ERDÉLYI \\ Dedicated to Peter Lax on the occasion of his 87th birthday
}

\begin{abstract}
We prove the right Lax-type inequality on subarcs of the unit circle of the complex plane for complex algebraic polynomials of degree $n$ having no zeros in the open unit disk. This is done by establishing the right Bernstein-Szegö-Videnskii type inequality for real trigonometric polynomials of degree at most $n$ on intervals shorter than the period. The paper is closely related to recent work by B. Nagy and V. Totik. In fact, their asymptotically sharp Bernstein-type inequality for complex algebraic polynomials of degree at most $n$ on subarcs of the unit circle is recaptured by using more elementary methods. Our discussion offers a somewhat new approach to see V.S. Videnskii's Bernstein and Markov type inequalities for trigonometric polynomials of degree at most $n$ on intervals shorter than a period, two classical polynomial inequalities published first in 1960. A new Riesz-Schur type inequality for trigonometric polynomials is also established. Combining this with Videnskii's Bernstein-type inequality gives Videnskii's Markov-type inequality immediately.
\end{abstract}

\section{INTRODUCTION}

Let $D$ be the open unit disk of the complex plane. Let $\partial D$ be the unit circle of the complex plane. Let $\mathcal{T}_{n}$ be the collection of all real trigonometric polynomials $Q$ of degree at most $n$ of the form

$$
Q(\tau)=a_{0}+\sum_{j=1}^{n}\left(a_{j} \cos (j \tau)+b_{j} \sin (j \tau)\right), \quad a_{j}, b_{j} \in \mathbb{R} .
$$

Let $\mathcal{T}_{n}^{c}$ be the collection of all complex trigonometric polynomials $Q$ of degree at most $n$ of the form

$$
Q(\tau)=a_{0}+\sum_{j=1}^{n}\left(a_{j} \cos (j \tau)+b_{j} \sin (j \tau)\right), \quad a_{j}, b_{j} \in \mathbb{C} .
$$

Key words and phrases. basic polynomial inequalities, Videnskii's Markov and Bernstein type inequalities for trigonometric polynomials on subintervals of the period, asymptotically sharp Bernstein and Lax type inequalities for complex algebraic polynomials on subarcs of the unit circle, the right Bernstein-Szegö and Riesz-Schur type inequalities for trigonometric polynomials on subintervals of the period..

2000 Mathematics Subject Classifications. 41A17 
Let $\mathcal{P}_{n}$ be the collection of all real algebraic polynomials $P$ of degree at most $n$ of the form

$$
P(z)=\sum_{j=0}^{n} a_{j} z^{j}, \quad a_{j} \in \mathbb{R} .
$$

Let $\mathcal{P}_{n}^{c}$ be the collection of all complex algebraic polynomials $P$ of degree at most $n$ of the form

$$
P(z)=\sum_{j=0}^{n} a_{j} z^{j}, \quad a_{j} \in \mathbb{C} .
$$

The following inequalities are due to Bernstein. We have

$$
\begin{aligned}
\max _{z \in \partial D}\left|P^{\prime}(z)\right| & \leq n \max _{z \in \partial D}|P(z)|, & P \in \mathcal{P}_{n}^{c}, \\
\max _{\tau \in[-\pi, \pi]}\left|Q^{\prime}(\tau)\right| & \leq n \max _{\tau \in[-\pi, \pi]}|Q(\tau)|, & Q \in \mathcal{T}_{n}^{c}, \\
\max _{x \in[-1,1]}\left|P^{\prime}(x) \sqrt{1-x^{2}}\right| & \leq n \max _{x \in[-1,1]}|P(x)|, & P \in \mathcal{P}_{n}^{c} .
\end{aligned}
$$

We remark that most likely M. Riesz was the first who published the second inequality above for all real trigonometric polynomials $Q \in \mathcal{T}_{n}$ without an extra factor 2 and with various complete (and elegant) proofs. However, the extention of this inequality from all real trigonometric polynomials $Q \in \mathcal{T}_{n}$ to all complex trigonometric polynomials $Q \in \mathcal{T}_{n}^{c}$ is a simple routine argument. The inequality

$$
\max _{\tau \in[-\pi, \pi]}\left(\left|Q^{\prime}(\tau)\right|^{2}+n^{2}|Q(\tau)|^{2}\right) \leq n^{2} \max _{\tau \in[-\pi, \pi]}|Q(\tau)|^{2}, \quad Q \in \mathcal{T}_{n},
$$

is often referred to as the Bernstein-Szegö inequality. Note that it is valid only for all real trigonometric polynomials $Q \in \mathcal{T}_{n}$ and not for all complex trigonometric polynomials $Q \in \mathcal{T}_{n}^{c}$. Markov's inequality asserts that

$$
\max _{x \in[-1,1]}\left|P^{\prime}(x)\right| \leq n^{2} \max _{x \in[-1,1]}|P(x)|, \quad Q \in \mathcal{P}_{n}^{c} .
$$

Books focusing on approximation theory contain the above mentioned inequalities with various proofs. See [16],[19], or Section 5.1 of [2], for example.

For $n \in \mathbb{N}, \omega \in(0, \pi]$, and $t \in(\omega, \omega)$, we define

$$
\begin{aligned}
B(n, \omega, t) & :=\frac{d}{d t}\left(-2 n \arccos \left(\frac{\sin (t / 2)}{\sin (\omega / 2)}\right)\right)=2 n\left(1-\left(\frac{\sin (t / 2)}{\sin (\omega / 2)}\right)^{2}\right)^{-1 / 2} \frac{\frac{1}{2} \cos (t / 2)}{\sin (\omega / 2)} \\
& =\frac{n \cos (t / 2)}{\sin (\omega / 2)}\left(1-\left(\frac{\sin (t / 2)}{\sin (\omega / 2)}\right)^{2}\right)^{-1 / 2} \\
& =\sqrt{2} n \cos (t / 2)(\cos t-\cos \omega)^{-1 / 2}
\end{aligned}
$$


Then

$$
(B(n, \omega, t))^{2}=\frac{2 n^{2} \cos ^{2}(t / 2)}{\cos t-\cos \omega}=n^{2} \frac{1+\cos t}{\cos t-\cos \omega}=n^{2}\left(\frac{1+\cos \omega}{\cos t-\cos \omega}+1\right) .
$$

Note that $B(n, \pi, t)=n$ for all $t \in(-\pi, \pi)$. The classical Bernstein inequality for trigonometric polynomials was extended by V.S. Videnskii, see e.g. [23] or E.19 of Section 5.1 on page 242 in [2]. He showed that

$$
\left|Q^{\prime}(t)\right| \leq B(n, \omega, t) \max _{\tau \in[-\omega, \omega]}|Q(\tau)|, \quad t \in(-\omega, \omega),
$$

for every $Q \in \mathcal{T}_{n}^{c}$. There is an extension of this to "trigonometric polynomials of halfinteger degree" in [24] where it is shown that

$$
\left|Q^{\prime}(t)\right| \leq B\left(n-\frac{1}{2}, \omega, t\right) \max _{\tau \in[-\omega, \omega]}|Q(\tau)|, \quad t \in(-\omega, \omega),
$$

for every "trigonometric polynomials of half-integer degree" $Q$ of the form

$$
Q(\tau)=\sum_{j=1}^{n}\left(a_{j} \cos \frac{(2 j-1) \tau}{2}+b_{j} \sin \frac{(2 j-1) \tau}{2}\right), \quad a_{j}, b_{j} \in \mathbb{R} .
$$

Bernstein-type inequalities for trigonometric polynomials in $L_{p}$ norms on subarcs of the unit circle were established by D. Lubinsky [14], C.K. Kobindarajah and D. Lubinsky [11], and T. Erdélyi [5].

In 1940 P. Lax [12] proved that

$$
\max _{\tau \in[-\pi, \pi]}\left|P^{\prime}\left(e^{i \tau}\right)\right| \leq \frac{n}{2} \max _{\tau \in[-\pi, \pi]}\left|P\left(e^{i \tau}\right)\right|
$$

for all polynomials $P \in \mathcal{P}_{n}^{c}$ having no zeros in the open unit disk $D$. Inequalities for polynomials with constraints are surveyed in $[2,4,6]$. Markov and Bernstein type inequalities for trigonometric polynomials on intervals shorter than the period are studied in $[3,7,8,9,10]$ under various constraints. Associated with an algebraic polynomial $P \in \mathcal{P}_{n}^{c}$ of the form

$$
P(z)=\sum_{j=0}^{n} a_{j} z^{j}, \quad a_{j} \in \mathbb{C},
$$

we introduce the polynomial $P^{*}$ defined by

$$
P^{*}(z):=\sum_{j=0}^{n} \bar{a}_{n-j} z^{j} .
$$

The algebraic polynomial $P$ of the above form is called conjugate reciprocal if $P=P^{*}$. In 1969 M.A. Malik [15] observed that

$$
\max _{\tau \in[-\pi, \pi]}\left(\left|P^{\prime}\left(e^{i \tau}\right)\right|+\left|P^{* \prime}\left(e^{i \tau}\right)\right|\right) \leq n \max _{\tau \in[-\pi, \pi]}\left|P\left(e^{i \tau}\right)\right|
$$


for every algebraic polynomial $P \in \mathcal{P}_{n}^{c}$. See also $[2,16]$. It was observed by A. Kroó, see e.g. E.16 c] on p. 438 in [2], that if $P \in \mathcal{P}_{n}^{c}$ has the property that $1 / \bar{a}$ is a zero of $P$ with multiplicity at least $k$ whenever $a \in D$ is a zero of $P$ with multiplicity $k$ (there is no restriction on the zeros of $P$ outside $D$ ), then

$$
\max _{\tau \in[-\pi, \pi]}\left|P^{\prime}\left(e^{i \tau}\right)\right| \leq \frac{n}{2} \max _{\tau \in[-\pi, \pi]}\left|P\left(e^{i \tau}\right)\right| .
$$

Both of the above observations generalize of Lax's inequality. We need to observe only that $\left|P^{\prime}(z)\right| \leq\left|P^{* \prime}(z)\right|$ for every $z \in \mathbb{C}$ with $|z|=1$. See e.g. page 438 of [2]. Lax-type inequalities for rational functions with fixed poles outside the closed unit circle were proved by X. Li, R.N. Mohapatra, and R.Z. Rodriguez [13]. This was discovered independently by P. Borwein and T. Erdélyi [2] (Theorem 7.11, p. 329) by using similar methods. Our first five theorems recapture some old results of Videnskii $[23,24]$ and some recent results of $\mathrm{V}$. Totik [20,21], and B. Nagy and V. Totik [18]. Our methods of proof are somewhat different and some of them may be viewed as somewhat more elementary. Note that Nagy and Totik $[17,18]$ and Totik $[20,21]$ establish more general results on Jordan curves as well as on closed and compact subsets of the period symmetric with respect to the origin using potential theoretic tools. Recently Totik [22] has extended even Videnskii's Markov-type inequality for trigonometric polynomials to unions of disjoint closed intervals. A number of interesting polynomial inequalities, including Remez-type inequalities, on Jordan curves are studied in the survey [1] by V.V. Andrievskii by using potential function theory and geometric function theory. Our Theorem 2.7 offers an extension of Malik's inequality to subarcs of the unit circle. This is based on our Theorem 2.6 that may be viewed as the special case of Theorem 2.7 dealing with conjugate reciprocal algebraic polynomials only. Our Theorem 2.8 is an extension of Lax's inequality to subarcs. Moreover, in Theorem 2.8 we assume only that $P \in \mathcal{P}_{n}^{c}$ satisfies the following: $1 / \bar{a}$ is a zero of $P$ with multiplicity at least $k$ whenever $a \in D$ is a zero of $P$ with multiplicity $k$ (there is no restriction on the zeros of $P$ outside $D$. Our Theorem 2.9 shows that Theorem 2.8 is asymptotically sharp. Theorems 2.12 and 2.13 establish the right Schur-type inequality for trigonometric polynomials of degree at most $n$. Despite their simplicity these inequalities do not seem to have appeared in the literature before. Theorem 2.13 is then used to recapture Videnskii's Markovtype inequality, stated as Theorem 2.11, from Videnskii's Bernstein-type inequality. This approach to prove Theorem 2.11 may be viewed as somewhat new.

\section{RESUlts}

Our first two theorems establish a Bernstein-Szegö-Videnskii type inequality for real trigonometric polynomials of integer and half-integer degree on intervals shorter than the period. They follow from the trigonometric Bernstein inequality and a general principle discovered by Totik [21]. However, in Section 3 we will present another self-contained proof of Theorems 2.1 and 2.2 below.

Theorem 2.1. Let $n \in \mathbb{N}$ and $\omega \in(0, \pi)$. We have

$$
\left|Q^{\prime}(t)\right|^{2}+(B(n, \omega, t))^{2}|Q(t)|^{2} \leq(B(n, \omega, t))^{2} \max _{\tau \in[-\omega, \omega]}|Q(\tau)|^{2}, \quad t \in(-\omega, \omega),
$$


for every $Q \in \mathcal{T}_{n}$.

A straightforward modification of the proof of Theorem 2.1 gives the following result.

Theorem 2.2. Let $n \in \mathbb{N}$ and $\omega \in(0, \pi)$. We have

$$
\left|Q^{\prime}(t)\right|^{2}+\left(B\left(n-\frac{1}{2}, \omega, t\right)\right)^{2}|Q(t)|^{2} \leq\left(B\left(n-\frac{1}{2}, \omega, t\right)\right)^{2} \max _{\tau \in[-\omega, \omega]}|Q(\tau)|^{2}, \quad t \in(-\omega, \omega),
$$

for all functions $Q$ of the form

$$
Q(\tau)=\sum_{j=1}^{n}\left(a_{j} \cos \frac{(2 j-1) \tau}{2}+b_{j} \sin \frac{(2 j-1) \tau}{2}\right), \quad a_{j}, b_{j} \in \mathbb{R} .
$$

It is routine now to derive the following Bernstein-type inequality from Theorems 2.1 and 2.2. This result is due to Videnskii [23,24], our approach to prove it is somewhat different from his.

Theorem 2.3. Let $n \in \mathbb{N}$ and $\omega \in(0, \pi)$. We have

$$
\left|R^{\prime}(t)\right| \leq B(n, \omega, t) \max _{\tau \in[-\omega, \omega]}|R(\tau)|, \quad t \in(-\omega, \omega),
$$

for all real trigonometric polynomials $R \in \mathcal{T}_{n}^{c}$. Furthermore, we have

$$
\left|R^{\prime}(t)\right| \leq B\left(n-\frac{1}{2}, \omega, t\right) \max _{\tau \in[-\omega, \omega]}|R(\tau)|, \quad t \in(-\omega, \omega),
$$

for all functions $R$ of the form

$$
R(\tau)=\sum_{j=1}^{n}\left(a_{j} \cos \frac{(2 j-1) \tau}{2}+b_{j} \sin \frac{(2 j-1) \tau}{2}\right), \quad a_{j}, b_{j} \in \mathbb{C} .
$$

Now we can easily prove the following Bernstein-type inequality for complex polynomials on a subarc of the unit circle. This is due to Nagy and Totik, see Theorem 1 in [18].

Theorem 2.4. Let $n \in \mathbb{N}$ and $\omega \in(0, \pi)$. We have

$$
\left|P^{\prime}\left(e^{i t}\right)\right| \leq \frac{1}{2}(B(n, \omega, t)+n) \max _{\tau \in[-\omega, \omega]}\left|P\left(e^{i \tau}\right)\right|, \quad t \in(-\omega, \omega),
$$

for every algebraic polynomial $P \in \mathcal{P}_{n}^{c}$.

The next theorem is stated as Theorem 2 in [18] and it shows that Theorem 2.4 is asymptotically sharp. Its proof is presented in [18] by using potential theoretic tools. In this paper we will show it by using quite an elementary approach. 
Theorem 2.5. For every fixed $\omega \in(0, \pi)$ and $t \in(-\omega, \omega)$ there are nonzero polynomials $P_{n} \in \mathcal{P}_{n}^{c}$ such that

$$
\left|P_{n}^{\prime}\left(e^{i t}\right)\right| \geq(1-o(1)) \frac{1}{2}(B(n, \omega, t)+n) \max _{\tau \in[-\omega, \omega]}\left|P_{n}\left(e^{i \tau}\right)\right|
$$

as $n$ tends to $\infty$.

Our next theorem improves the Nagy-Totik inequality for conjugate reciprocal polynomials of degree $n$.

Theorem 2.6. Let $n \in \mathbb{N}$ and $\omega \in(0, \pi)$. We have

$$
\left|P^{\prime}\left(e^{i t}\right)\right| \leq \frac{1}{2} B(n, \omega, t) \max _{\tau \in[-\omega, \omega]}\left|P\left(e^{i \tau}\right)\right|
$$

for every conjugate reciprocal algebraic polynomial $P \in \mathcal{P}_{n}^{c}$ of degree $n$ and for every $t \in(-\omega, \omega)$.

The above theorem follows simply from the Malik-type inequality below.

Theorem 2.7. Let $n \in \mathbb{N}$ and $\omega \in(0, \pi)$. We have

$$
\left|P^{\prime}\left(e^{i t}\right)\right|+\left|P^{* \prime}\left(e^{i t}\right)\right| \leq B(n, \omega, t) \max _{\tau \in[-\omega, \omega]}\left|P\left(e^{i \tau}\right)\right|
$$

for every $P \in \mathcal{P}_{n}^{c}$ of degree $n$ and for every $t \in(-\omega, \omega)$.

Our next result is an extention of Lax's polynomial inequality to subarcs of the unit circle.

Theorem 2.8. Let $n \in \mathbb{N}$ and $\omega \in(0, \pi)$. Suppose $P \in \mathcal{P}_{n}^{c}$ has the property that $1 / \bar{a}$ is a zero of $P$ with multiplicity at least $k$ whenever $a \in D$ is a zero of $P$ with multiplicity $k$ (there is no restriction on the zeros of $P$ outside $D$. We have

$$
\left|P^{\prime}\left(e^{i t}\right)\right| \leq \frac{1}{2} B(n, \omega, t) \max _{\tau \in[-\omega, \omega]}\left|P\left(e^{i \tau}\right)\right|
$$

for every $t \in(-\omega, \omega)$.

Our next theorem tells us that the Lax-type inequality of Theorem 2.9 is asymptotically sharp.

Theorem 2.9. For every fixed $\omega \in(0, \pi)$ and $t \in(-\omega, \omega)$ there are nonzero polynomials $P_{n} \in \mathcal{P}_{n}^{c}$ with no zeros in the open unit disk $D$ such that

$$
\left.\left|P_{n}^{\prime}\left(e^{i t}\right)\right| \geq(1-o(1)) \frac{1}{2} B(n, \omega, t)\right) \max _{\tau \in[-\omega, \omega]}\left|P_{n}\left(e^{i \tau}\right)\right|
$$

as $n$ tends to $\infty$.

In the proof of Theorem 2.8 we need the result below. 
Lemma 2.10. If $k \geq 0$ and $u \geq k$ are integers,

$$
Q(z)=\prod_{j=1}^{k}\left(z-r_{j} e^{i \varphi_{j}}\right)\left(z-r_{j}^{-1} e^{-i \varphi_{j}}\right) \prod_{j=k+1}^{u}\left(z-r_{j} e^{i \varphi_{j}}\right)
$$

with

$$
r_{j} \in(0,1), \varphi_{j} \in \mathbb{R}, \quad j=1,2, \ldots, u,
$$

then $Q^{\prime}\left(e^{i \theta}\right) \neq 0$ for every $\theta \in \mathbb{R}$.

Our next theorem states Videnskii's Markov-type inequality [23] on intervals shorter than the period. An outline of its proof may also be found on pages 243-245 of [2]. The main ideas of the proof are quite similar to those used in the proof of Theorem 2.1. We remark that it is routine to extend the result below to the class $\mathcal{T}_{n}^{c}$ by using the method in the proof of Theorem 2.3.

Theorem 2.11. Let $n \in \mathbb{N}$ and $\omega \in(0, \pi)$. We have

$$
\max _{\tau \in[-\omega, \omega]}\left|R^{\prime}(\tau)\right| \leq U_{n}^{\prime}(\omega) \max _{\tau \in[-\omega, \omega]}|Q(\tau)|=2 n^{2} \cot (\omega / 2) \max _{\tau \in[-\omega, \omega]}|Q(\tau)|
$$

for every $R \in \mathcal{T}_{n}$ and $2 n>\left(3 \tan ^{2}(\omega / 2)+1\right)^{1 / 2}$, and equality holds if and only if $R=c U_{n}$, where $U_{n} \in \mathcal{T}_{n}$ is of the form

$$
U_{n}(\tau)=\cos \left(2 n \arccos \left(\frac{\sin (\tau / 2)}{\sin (\omega / 2}\right)\right), \quad \tau \in[-\omega, \omega],
$$

and $c \in \mathbb{R}$.

Our next result is a Riesz-Schur type inequality for trigonometric polynomials on intervals shorter than the period. This may be viewed as a new result. To formulate it, associated with $n \in \mathbb{N}$ and $\omega \in(0, \pi)$ let

$$
\tau_{k}:=2 \arcsin \left(\left(\sin \frac{\omega}{2}\right) \cos \frac{(4 n-2 k+1) \pi}{4 n}\right), \quad k=1,2, \ldots, 2 n,
$$

be the zeros of $U_{n} \in \mathcal{T}_{n}$ defined in Theorem 2.11. Observe that

$$
-\omega<\tau_{1}<\tau_{2}<\cdots<\tau_{2 n}<\omega .
$$

Theorem 2.12. Let $n \in \mathbb{N}$ and $\omega \in(0, \pi)$. Assume that $Q \in \mathcal{T}_{n}$,

$$
\left|Q\left(\tau_{k}\right)\right| \leq B\left(n, \omega, \tau_{k}\right), \quad k=1,2, \ldots, 2 n,
$$

and

$$
|Q( \pm \omega)| \leq 2 n^{2} \cot (\omega / 2)
$$

Then

$$
|Q(\tau)| \leq\left|U_{n}^{\prime}(\tau)\right|, \quad \tau \in\left[-\omega, \tau_{1}\right] \cup\left[\tau_{2 n}, \omega\right]
$$

Next we state a simple consequence of Theorem 2.12. It can be used to obtain Videnskii's Markov-type inequality from Videnskii's Bernstein-type inequality for trigonometric polynomials on intervals shorter than the period. The theorem below may be viewed as a new result as well. 
Theorem 2.13. Let $n \in \mathbb{N}$ and $\omega \in(0, \pi)$. Assume that $Q \in \mathcal{T}_{n}$,

$$
\begin{gathered}
|Q(\tau)| \leq B(n, \omega, \tau), \quad \tau \in(-\omega, \omega) \\
|Q( \pm \omega)| \leq 2 n^{2} \cot (\omega / 2) \quad \text { and } \quad 2 n>\left(3 \tan ^{2}(\omega / 2)+1\right)^{1 / 2}
\end{gathered}
$$

Then

$$
\max _{\tau \in[-\omega, \omega]}|Q(\tau)| \leq 2 n^{2} \cot (\omega / 2)
$$

To deduce Theorem 2.11 from Theorem 2.3 applied to $R \in \mathcal{T}_{n}$ and Theorem 2.13 applied to $Q=R^{\prime} \in \mathcal{T}_{n}$ we need to see only that the assumption

$$
|Q( \pm \omega)|=\left|R^{\prime}( \pm \omega)\right| \leq\left|U_{n}^{\prime}( \pm \omega)\right|=2 n^{2} \cot (\omega / 2)
$$

holds for all $R \in \mathcal{T}_{n}$ satisfying

$$
\max _{\tau \in[-\omega, \omega]}|R(\tau)|=1
$$

However, this is a simple observation as the extremal polynomial $R^{*} \in \mathcal{T}_{n}$ satisfying

$$
\frac{\left|R^{* \prime}( \pm \omega)\right|}{\max _{\tau \in[-\omega, \omega]}\left|R^{*}(\tau)\right|}=\sup _{0 \neq R \in \mathcal{T}_{n}} \frac{\left|R^{\prime}( \pm \omega)\right|}{\max _{\tau \in[-\omega, \omega]}|R(\tau)|}
$$

can be characterized by equioscillating $2 n+1$ times on the interval $[-\omega, \omega]$, and hence it can be identified as $R^{*}= \pm U_{n}$.

We close this section by recalling the following Riesz-Schur type inequality proved in [8] for trigonometric polynomials on intervals shorter than the period. However, although it is a sharp result, it is not the "right" Riesz-Schur type inequality to derive Videnskii's Markov-type inequality from Videnskii's Bernstein-type inequality for trigonometric polynomials on intervals shorter than the period.

Theorem 2.14. Let $n \in \mathbb{N}$ and $\omega \in(0, \pi)$. We have

$$
A_{n}(\omega):=\sup _{0 \neq Q \in \mathcal{T}_{n}} \frac{\max _{\tau \in[-\omega, \omega]}|Q(\tau)|}{\max _{\tau \in[-\omega, \omega]}\left|Q(\tau)\left(\frac{1}{2}(\cos \tau-\cos \omega)\right)^{1 / 2}\right|}=\frac{2 n+1}{\sin (\omega / 2)}
$$

and the supremum is attained if and only if

$$
Q(\tau)=c \frac{\cos \left((2 n+1) \arccos \left(\frac{\sin (\tau / 2)}{\sin (\omega / 2}\right)\right)}{(\cos \tau-\cos \omega)^{1 / 2}}
$$

with some $0 \neq c \in \mathbb{R}$. 


\section{ProOFs}

Proof of Theorem 2.1. Let $t \in(-\omega, \omega)$ and $n \in \mathbb{N}$ be fixed. A simple compactness argument shows that there is a trigonometric polynomial $Q^{*} \in \mathcal{T}_{n}$ such that

$$
\left|Q^{* \prime}(t)\right|^{2}+(B(n, \omega, t))^{2}\left|Q^{*}(t)\right|^{2}=\sup _{Q \in \mathcal{T}_{n}}\left(\left|Q^{\prime}(t)\right|^{2}+(B(n, \omega, t))^{2}|Q(t)|^{2}\right),
$$

where the supremum is taken for all $Q \in \mathcal{T}_{n}$ with

$$
\max _{\tau \in[-\omega, \omega]}|Q(\tau)|=1
$$

It can be shown by a standard variational method that $Q^{*}$ equioscillates in $[-\omega, \omega]$ at least $2 n$ times. That is, there are

$$
-\omega \leq \tau_{1}<\tau_{2}<\cdots<\tau_{2 n} \leq \omega
$$

such that

$$
Q^{*}\left(\tau_{j}\right)= \pm(-1)^{j}, \quad j=1,2, \ldots, 2 n
$$

To see this let

$$
E:=\left\{\tau \in[-\omega, \omega]:\left|Q^{*}(\tau)\right|=1\right\} .
$$

There are $E_{1}, E_{2}, \ldots, E_{m}$ such that

$$
E=\bigcup_{j=1}^{m} E_{j}, \quad \max E_{j}<\min E_{j+1}, \quad j=1,2, \ldots, m-1,
$$

and

$$
Q^{*}(\tau)= \pm(-1)^{j}, \quad \tau \in E_{j}, \quad j=1,2, \ldots, m .
$$

Since $t \in(-\omega, \omega)$ and $Q^{* \prime}(t) \neq 0$, we have $t \notin E$. We pick

$$
\alpha_{j} \in\left(\max E_{j}, \min E_{j+1}\right), \quad j=1,2, \ldots, m-1 .
$$

If $m \leq 2 n-1$, then we can choose a trigonometric polynomial $R \in \mathcal{T}_{n}$ of the form

$$
R(\tau)=c\left(\prod_{j=1}^{m-1} \sin \frac{\tau-\alpha_{j}}{2}\right) \sin ^{2} \frac{\tau-t}{2} \sin ^{2 n-1-m} \frac{\tau-\pi}{2}
$$

where the constant $c \neq 0$ is chosen so that $R(\tau) Q^{*}(\tau)>0$ for $\tau \in E_{1}$, and hence

$$
R(\tau) Q^{*}(\tau)>0, \quad \tau \in E_{j}, \quad j=1,2, \ldots, m .
$$

Let $Q_{\varepsilon}:=c_{\varepsilon}\left(Q^{*}-\varepsilon R\right)$, where the constant $c_{\varepsilon}>0$ is chosen so that

$$
\max _{\tau \in[-\omega, \omega]}\left|Q_{\varepsilon}(\tau)\right|=1
$$


If $\varepsilon>0$ is sufficiently small then $c_{\varepsilon}>1$, and $Q_{\varepsilon} \in \mathcal{T}_{n}$ contradicts the extremal property of $Q^{*}$. Hence $m \geq 2 n$, that is, $Q^{*}$ equioscillates in $[-\omega, \omega]$ at least $2 n$ times, as we stated.

Now it is easy to see that one of the two cases below holds.

Case 1. $Q^{*}$ equioscillates $2 n+1$ times on a larger interval $[-\widetilde{\omega}, \omega]$ or $[-\omega, \widetilde{\omega}]$ with some $\widetilde{\omega} \in[\omega, 2 \pi-\omega)$.

Case 2. $Q^{*}$ equioscillates $2 n$ times on a period of length $2 \pi$.

In Case 1 without loss of generality we may assume that $Q^{*}$ equioscillates $2 n+1$ times on $[-\omega, \widetilde{\omega}]$ with some $\widetilde{\omega} \in[\omega, 2 \pi-\omega)$, the other case is analogous. Thus, there are

$$
-\omega=\tau_{0}<\tau_{1}<\cdots<\tau_{2 n}=\widetilde{\omega}
$$

such that

$$
Q^{*}\left(\tau_{j}\right)= \pm(-1)^{j}, \quad j=1,2, \ldots, 2 n .
$$

Then it is a routine argument to identify $Q^{*}$ as

$$
Q^{*}(\tau)= \pm \cos \left(2 n \arccos \left(\frac{\sin ((\tau-\alpha) / 2))}{\sin (\beta / 2)}\right)\right), \quad \tau \in[-\omega, \omega] \subset[-\omega, \widetilde{\omega}],
$$

with

$$
\alpha:=\frac{\widetilde{\omega}-\omega}{2} \quad \text { and } \quad \beta:=\frac{\widetilde{\omega}+\omega}{2}=\omega+\alpha<\pi .
$$

Therefore

$$
\left|Q^{* \prime}(t)\right|^{2}+(B(n, \beta, t-\alpha))^{2}\left|Q^{*}(t)\right|^{2}=(B(n, \beta, t-\alpha))^{2} .
$$

Elementary calculus shows that

$$
(B(n, \beta, t-\alpha))^{2}=(B(n, \omega+\alpha, t-\alpha))^{2} \leq(B(n, \omega, t))^{2}, \quad \alpha \in[0, \pi-\omega) .
$$

To see this we have to show that

$$
\frac{1+\cos (\omega+\alpha)}{\cos (t-\alpha)-\cos (\omega+\alpha)} \leq \frac{1+\cos \omega}{\cos t-\cos \omega},
$$

that is,

$$
\frac{1+\cos (\omega+\alpha)}{1+\cos \omega} \leq \frac{\cos (t-\alpha)-\cos (\omega+\alpha)}{\cos t-\cos \omega} .
$$

However,

$$
\begin{aligned}
\frac{\cos (t-\alpha)-\cos (\omega+\alpha)}{\cos t-\cos \omega} & =\frac{2 \sin \left(\frac{1}{2}(\omega+t)\right) \sin \left(\frac{1}{2}(\omega-t)+\alpha\right)}{2 \sin \left(\frac{1}{2}(\omega+t)\right) \sin \left(\frac{1}{2}(\omega-t)\right)} \\
& =\frac{\sin \left(\left(\frac{1}{2}(\omega-t)+\alpha\right)\right.}{\sin \left(\frac{1}{2}(\omega-t)\right)} .
\end{aligned}
$$


Let

$$
f(\tau):=\frac{\sin (\tau+\alpha)}{\sin \tau}, \quad \tau \in(0, \pi)
$$

Then

$$
f^{\prime}(\tau)=\frac{-\sin \alpha}{\sin ^{2} \tau},
$$

hence $f$ is decreasing on $(0, \pi)$. Therefore $t \in(-\omega, \omega)$ implies $f(\omega) \leq f\left(\frac{1}{2}(\omega-t)\right)$, that is,

$$
\frac{\sin (\omega+\alpha)}{\sin \omega} \leq \frac{\sin \left(\frac{1}{2}(\omega-t)+\alpha\right)}{\sin \left(\frac{1}{2}(\omega-t)\right)}
$$

Combining this with (3.5) we deduce that in order to prove (3.4) we have to show only

$$
\frac{1+\cos (\omega+\alpha)}{1+\cos \omega} \leq \frac{\sin (\omega+\alpha)}{\sin \omega}
$$

However, this is equivalent to

$$
\tan \left(\frac{1}{2}(\omega+\alpha)\right) \geq \tan \left(\frac{1}{2} \omega\right)
$$

which obviously holds since $\omega / 2 \leq(\omega+\alpha) / 2<\pi / 2$. So (3.3) is justified.

Now (3.1) and (3.2) give that

$$
\begin{aligned}
& \left|Q^{* \prime}(t)\right|^{2}+(B(n, \omega, t))^{2}\left|Q^{*}(t)\right|^{2} \\
= & \left|Q^{* \prime}(t)\right|^{2}+(B(n, \beta, t-\alpha))^{2}\left|Q^{*}(t)\right|^{2}+\left((B(n, \omega, t))^{2}-(B(n, \beta, t-\alpha))^{2}\right)\left|Q^{*}(t)\right|^{2} \\
\leq & (B(n, \beta, t-\alpha))^{2}+\left((B(n, \omega, t))^{2}-(B(n, \beta, t-\alpha))^{2}\right) \\
= & (B(n, \omega, t))^{2} .
\end{aligned}
$$

This finishes the proof in Case 1.

In Case 2, first observe that

$$
(B(n, \omega, t))^{2}=n^{2}\left(\frac{1+\cos \omega}{\cos t-\cos \omega}+1\right) \geq n^{2} .
$$

Hence the Bernstein-Szegő inequality implies that

$$
\begin{aligned}
& \left|Q^{* \prime}(t)\right|^{2}+(B(n, \omega, t))^{2}\left|Q^{*}(t)\right|^{2} \\
= & \left|Q^{* \prime}(t)\right|^{2}+n^{2}\left|Q^{*}(t)\right|^{2}+\left((B(n, \omega, t))^{2}-n^{2}\right)\left|Q^{*}(t)\right|^{2} \\
\leq & n^{2}+\left((B(n, \omega, t))^{2}-n^{2}\right) \\
= & (B(n, \omega, t))^{2} .
\end{aligned}
$$

(In fact, it is easy to see that in this case $Q^{*}$ is of the form $Q^{*}(x)=\sin (n(x-\gamma)$ ) with some $\gamma \in \mathbb{R}$, so a simple calculus instead of the Bernstein-Szegö inequality already implies the above inequality.) This finishes the proof in Case 2. 
Proof of Theorem 2.3. We prove only the first statement, the second one can be verified in the same way. If $R \in \mathcal{T}_{n}^{c}$ is a trigonometric polynomial of degree at most $n$ and $t \in(-\omega, \omega)$, then pick $\alpha \in \mathbb{C}$ with $|\alpha|=1$ so that $\alpha R^{\prime}(t)=\left|R^{\prime}(t)\right|$. Now applying Theorem 2.1 to the real trigonometric polynomial $Q \in \mathcal{T}_{n}$ defined as $Q(\tau):=\operatorname{Re}(\alpha R(\tau))$, we get theorem.

Proof of Theorem 2.4. Let $P \in \mathcal{P}_{n}^{c}$. We introduce $R$ as

$$
R(\tau)=e^{-i n \tau / 2} P\left(e^{i \tau}\right)
$$

If $n=2 m$ is even, then $R \in \mathcal{T}_{m}^{c}$, while if $n=2 m-1$ is odd, then $R$ is a function of the form

$$
R(\tau)=\sum_{j=1}^{n}\left(a_{j} \cos \frac{(2 j-1) \tau}{2}+b_{j} \sin \frac{(2 j-1) \tau}{2}\right), \quad a_{j}, b_{j} \in \mathbb{C} .
$$

In both cases we have

$$
R^{\prime}(t)=e^{-i n t / 2}(-i n / 2) P\left(e^{i t}\right)+e^{-i n t / 2} P^{\prime}\left(e^{i t}\right) e^{i t} i
$$

and hence

$$
\left|P^{\prime}\left(e^{i t}\right)\right| \leq\left|R^{\prime}(t)\right|+\frac{n}{2}\left|P\left(e^{i t}\right)\right| .
$$

The theorem now follows from Theorem 2.3.

Proof of Theorem 2.5. Let $0<2 \varepsilon<\pi-\omega$ and $\widetilde{\omega}:=\omega+2 \varepsilon$. We define

$$
U_{k}(\tau):=\cos \left(2 k \arccos \left(\frac{\sin (\tau / 2)}{\sin (\widetilde{\omega} / 2}\right)\right), \quad \tau \in[-\widetilde{\omega}, \widetilde{\omega}]
$$

and

$$
V_{k}(\tau):=\sin \left(2 k \arccos \left(\frac{\sin (\tau / 2)}{\sin (\widetilde{\omega} / 2}\right)\right), \quad \tau \in[-\widetilde{\omega}, \widetilde{\omega}] .
$$

Observe that $U_{k} \in \mathcal{T}_{k}$, and each of the intervals between consecutive extreme points of $U_{k}$ on $[-\widetilde{\omega}, \widetilde{\omega}]$ contains at most one extreme point of $T_{k}$ defined by $T_{k}(\tau)=\sin (k \tau)$ on the period, otherwise either $U_{k}-T_{k} \in \mathcal{T}_{k}$ or $U_{k}+T_{k} \in \mathcal{T}_{k}$ would have at least $2 k+1$ zeros in $[-\widetilde{\omega}, \widetilde{\omega}]$ counted with multiplicities, a contradiction. Hence, the distance between any two consecutive extreme points of $U_{k}$ is at most $\pi / k$. Let $n=2 m, m=k+u, u=o(m)$. We define $P_{n} \in \mathcal{P}_{n}^{c}$ by

$$
e^{-i m \tau} P_{n}\left(e^{i \tau}\right):=Q_{m}(\tau)+i R_{m}(\tau)
$$

with $Q_{m}:=U_{k} \in \mathcal{T}_{m}, R_{m}:=V_{k} S_{u} \in \mathcal{T}_{m}$, where $S_{u}$ is defined by

$$
S_{u}(\tau):=H_{u}\left(\frac{\sin (\tau / 2)}{\sin (\widetilde{\omega} / 2)}\right), \quad \tau \in[-\widetilde{\omega}, \widetilde{\omega}],
$$

with an odd $H_{u} \in \mathcal{P}_{2 u-1}$. It is easy to see that the fact that $H_{u} \in \mathcal{P}_{2 u-1}$ is odd ensures that both $Q_{m}$ and $R_{m}$ are in $\mathcal{T}_{m}$, indeed, and hence $P_{n} \in \mathcal{P}_{n}^{c}$. Let $t \in(-\omega, \omega)$ be fixed. Without loss of generality we may assume that $t \in[0, \omega)$. Let

$$
a:=a_{t, \varepsilon, k}:=\min \left\{\tau \geq t: U_{k}(\tau)=0, \tau \in(-\widetilde{\omega}, \widetilde{\omega}) \backslash(-\varepsilon, \varepsilon)\right\} .
$$


As the distance between any two consecutive extreme points of $U_{k}$ is at most $\pi / k$, it is easy to see that $|t-a| \leq 2 \pi / k+\varepsilon$. Note that $U_{k}(a)=0$ implies $\left|V_{k}(a)\right|=1$. Let $L:=L_{\varepsilon}$ be the piecewise linear continuous function defined on $[-\widetilde{\omega}, \widetilde{\omega}]$ taking the value 1 on $[-\widetilde{\omega},-\varepsilon]$, and the value -1 on $[\varepsilon, \widetilde{\omega}]$. Using the Weierstrass Approximation Theorem we can pick the odd polynomial $H_{u} \in \mathcal{P}_{2 u-1}$ so that

$$
\max _{\tau \in[-\widetilde{\omega}, \widetilde{\omega}]}\left|S_{u}(\tau)-L(\tau)\right| \leq \varepsilon
$$

We have

$$
\left|S_{u}(a)+1\right| \leq \varepsilon \quad \text { and } \quad \max _{\tau \in[-\widetilde{\omega}, \widetilde{\omega}]}\left|S_{u}(\tau)\right| \leq 1+\varepsilon
$$

It follows that

$$
\begin{aligned}
\left|P_{n}\left(e^{i \tau}\right)\right|^{2} & =\left|Q_{m}(\tau)\right|^{2}+\left|R_{m}(\tau)\right|^{2}=\left|U_{k}(\tau)\right|^{2}+\left|V_{k}(\tau)\right|^{2}\left|S_{u}(\tau)\right|^{2} \\
& \leq(1+\varepsilon)^{2}\left(\left|U_{k}(\tau)\right|^{2}+\left|V_{k}(\tau)\right|^{2}\right) \leq(1+\varepsilon)^{2}, \quad \tau \in[-\widetilde{\omega}, \widetilde{\omega}],
\end{aligned}
$$

that is,

$$
\max _{\tau \in[-\widetilde{\omega}, \widetilde{\omega}]}\left|P_{n}\left(e^{i \tau}\right)\right| \leq(1+\varepsilon)
$$

Also, using $\left|S_{u}(a)+1\right| \leq \varepsilon$ and $\left|V_{k}(a)\right|=1$, we easily deduce that

$$
\begin{aligned}
i e^{i \tau} P_{n}^{\prime}\left(e^{i \tau}\right) & =\frac{d}{d \tau} P_{n}\left(e^{i \tau}\right)=\frac{d}{d \tau}\left(e^{i m \tau}\left(Q_{m}(\tau)+i R_{m}(\tau)\right)\right) \\
& =e^{i m \tau}\left(Q_{m}^{\prime}(\tau)+i R_{m}^{\prime}(\tau)+i m\left(Q_{m}(\tau)+i R_{m}(\tau)\right)\right) \\
& =e^{i m \tau}\left(\left(Q_{m}^{\prime}(\tau)-m R_{m}(\tau)\right)+i\left(R_{m}^{\prime}(\tau)+m\left(Q_{m}(\tau)\right)\right) .\right.
\end{aligned}
$$

This, together with $\left|S_{u}(a)+1\right| \leq \varepsilon$ and $\left|V_{k}(a)\right|=1$, implies that

$$
\begin{aligned}
\left|P_{n}^{\prime}\left(e^{i a}\right)\right| & =\left|e^{-i m a} i e^{i a} P_{n}^{\prime}\left(e^{i a}\right)\right| \geq\left|\operatorname{Re}\left(e^{-i m a} i e^{i a} P_{n}^{\prime}\left(e^{i a}\right)\right)\right| \\
& \geq\left|Q_{m}^{\prime}(a)-m R_{m}(a)\right|=\left|U_{k}^{\prime}(a)-m V_{k}(a) S_{u}(a)\right| \\
& \left.=\mid B(k, a, \widetilde{\omega}) V_{k}(a)+m V_{k}(a)-m\left(S_{u}(a)+1\right) V_{k}(a)\right) \mid \\
& \geq(B(k, a, \widetilde{\omega})+m)\left|V_{k}(a)\right|-m\left|S_{u}(a)+1\right|\left|V_{k}(a)\right| \\
& =B(k, a, \widetilde{\omega})+m-m \varepsilon .
\end{aligned}
$$

Now let $P_{n, t} \in \mathcal{P}_{n}^{c}$ be defined by

$$
P_{n, t}\left(e^{i \tau}\right):=P_{n}\left(e^{i(\tau+a-t)}\right), \quad t \in \tau \in \mathbb{R}
$$

If $\varepsilon>0$ is sufficiently small and $k$ and $n$ are sufficiently large, then

$$
\frac{B(k, a, \widetilde{\omega})+m-m \varepsilon}{B(k, t, \widetilde{\omega})+m} \text { is as close to } 1 \text { as we wish, }
$$


and $|t-a| \leq 2 \pi / k+\varepsilon<2 \varepsilon$ and (3.6) imply that

$$
\max _{\tau \in[-\omega, \omega]}\left|P_{n, t}\left(e^{i \tau}\right)\right| \leq \max _{\tau \in[-\widetilde{\omega}, \widetilde{\omega}]}\left|P_{n}\left(e^{i \tau}\right)\right| \leq(1+\varepsilon)
$$

Observe that (3.8) and (3.7) imply

$$
\left|P_{n, t}^{\prime}\left(e^{i t}\right)\right|=\left|P_{n}^{\prime}\left(e^{i a}\right)\right|=B(k, a, \widetilde{\omega}) .
$$

Combining (3.9), (3.10), and (3.11), and recalling that $n=2 m, m=k+u$, and $u=o(m)$, we get the theorem.

Proof of Theorem 2.6. If the algebraic polynomial $P \in \mathcal{P}_{n}^{c}$ of degree $n$ is conjugate reciprocal then $Q$ defined by

$$
Q(\tau)=e^{-i n \tau / 2} P\left(e^{i \tau}\right)
$$

is a real trigonometric polynomial of degree $n / 2$. Hence we can apply the Bernstein-SzegöVidenskii inequality of Theorem 2.1 (when $n$ is even) or Theorem 2.2 (when $n$ is odd) to $Q_{n}$ to obtain

$$
\begin{aligned}
\left|P^{\prime}\left(e^{i t}\right)\right| & =\left|i e^{i t} P^{\prime}\left(e^{i t}\right)\right|=\left|Q^{\prime}(t) e^{i n t / 2}+(i n / 2) e^{i n t / 2} Q(t)\right|=\left|Q^{\prime}(t)+(i n / 2) Q(t)\right| \\
& \leq B(n / 2, \omega, t) \max _{\tau \in[-\omega, \omega]}|Q(\tau)|=\frac{1}{2} B(n, \omega, t) \max _{\tau \in[-\omega, \omega]}|Q(\tau)|
\end{aligned}
$$

Proof of Theorem 2.7. Let $P \in \mathcal{P}_{n}^{c}$. Let $c=e^{i \gamma}$, where $\gamma \in \mathbb{R}$ will be chosen later. We define $R:=c P$. Then $R^{*}=\bar{c} P_{n}^{*}$. Observe that $S:=R+R^{*}$ satisfies $S=S^{*}$, and hence it is a conjugate reciprocal algebraic polynomial of degree at most $n$. Observe that $\overline{R^{*}(z)}=z^{-n} R(z)$ and hence $\left|R^{*}(z)\right|=|R(z)|$ for all $z \in \partial D$. Therefore

$$
\max _{\tau \in[-\omega, \omega]}\left|S\left(e^{i \tau}\right)\right| \leq 2 \max _{\tau \in[-\omega, \omega]}\left|R\left(e^{i \tau}\right)\right|=2 \max _{\tau \in[-\omega, \omega]}\left|P\left(e^{i \tau}\right)\right|
$$

Using Theorem 2.6 with $S$ we conclude that

$$
\begin{aligned}
\left|\left(c P^{\prime}+\bar{c} P^{* \prime}\right)\left(e^{i t}\right)\right| & =\left|\left(R^{\prime}+R^{* \prime}\right)\left(e^{i t}\right)\right|=\left|S^{\prime}\left(e^{i t}\right)\right| \\
& \leq \frac{1}{2} B(n, \omega, t) \max _{\tau \in[-\omega, \omega]}\left|S\left(e^{i \tau}\right)\right| \\
& \leq B(n, \omega, t) \max _{\tau \in[-\omega, \omega]}\left|P\left(e^{i \tau}\right)\right| .
\end{aligned}
$$

Now we choose $c=e^{i \gamma}$ with $\gamma \in \mathbb{R}$ so that

$$
\gamma:=\frac{1}{2} \arg \left(\frac{P^{* \prime}\left(e^{i t}\right)}{P^{\prime}\left(e^{i t}\right)}\right)
$$


if $P^{\prime}\left(e^{i t}\right) P^{* \prime}\left(e^{i t}\right) \neq 0$, and $\gamma \in \mathbb{R}$ can be arbitrary if $P^{\prime}\left(e^{i t}\right) P^{* \prime}\left(e^{i t}\right)=0$. We conclude that

$$
\begin{aligned}
\left|P^{\prime}\left(e^{i t}\right)\right|+\left|P^{* \prime}\left(e^{i t}\right)\right| & =\left|\left(c P^{\prime}+\bar{c} P^{* \prime}\right)\left(e^{i t}\right)\right|=\left|\left(R^{\prime}+R^{* \prime}\right)\left(e^{i t}\right)\right|=\left|S^{\prime}\left(e^{i t}\right)\right| \\
& \leq B(n, \omega, t) \max _{\tau \in[-\omega, \omega]}\left|S\left(e^{i \tau}\right)\right| \\
& \leq B(n, \omega, t) \max _{\tau \in[-\omega, \omega]}\left|P\left(e^{i \tau}\right)\right| .
\end{aligned}
$$

Proof of Theorem 2.8. This follows from Theorem 2.7. We need to observe only that $\left|P^{\prime}(z)\right| \leq\left|P^{* \prime}(z)\right|$ for every $z \in \partial D$. This statement may be found on page 438 of [2], as a hint to an exercise. It may be proved as follows. Let $c \in \mathbb{C}$ with $|c|<1$. Without loss of generality we may assume that $P$ has no zeros on the unit circle; and then the statement in the general case follows by a continuity argument. Observe that $|P(z)|=\left|P^{*}(z)\right|$ for all $z \in \partial D$, and hence Rouche's Theorem implies that $P^{*}$ and $Q:=P^{*}-c P$ have the same number of zeros in the open unit disk $D$ (by counting multiplicities). Now observe that if $P$ satisfies the assumption of the theorem, then $Q:=P^{*}-c P$ is of the form specified in Theorem 2.10. Hence $Q^{\prime}:=P^{* \prime}-c P^{\prime}$ does not vanish on the unit circle, and $\left|P^{\prime}(z)\right| \leq\left|P^{* \prime}(z)\right|$ follows for all $z \in \partial D$, as we stated. This finishes the proof of the theorem.

Proof of Theorem 2.9. Let $n=2 m$ and let $T_{m} \in \mathcal{T}_{m}$ be defined by

$$
T_{m}(\tau)=\cos \left(2 m \arccos \left(\frac{\sin (\tau / 2)}{\sin (\omega / 2)}\right)\right), \quad \tau \in[-\omega, \omega] .
$$

Let $P_{n} \in \mathcal{P}_{n}$ be defined by

$$
P_{n}\left(e^{i \tau}\right)=e^{i m \tau} T_{m}(\tau)
$$

Then $P_{n}$ has all its zeros on the unit circle, in fact on the subarc $\left\{e^{i \tau}: \tau \in[-\omega, \omega]\right\}$ of the unit circle, and

$$
\left|P_{n}^{\prime}\left(e^{i t}\right)\right|=B(m, \omega, t)=\frac{1}{2} B(n, \omega, t) \max _{\tau \in[-\omega, \omega]}\left|P_{n}\left(e^{i \tau}\right)\right|
$$

at every $t$ for which $P\left(e^{i t}\right)=0$. It is left to the reader to see how the above observation implies that Theorem 2.8 is asymptotically sharp for all $\omega \in(0, \pi)$ and $t \in(-\omega, \omega)$ as $n$ tends to $\infty$.

Proof of Lemma 2.10. A simple calculation shows that

$$
\begin{aligned}
\operatorname{Re}\left(\frac{e^{i \theta} Q^{\prime}\left(e^{i \theta}\right)}{Q\left(e^{i \theta}\right)}\right) & =\sum_{j=1}^{k} \frac{\left(r_{j}+r_{j}^{-1}-2 \cos \left(\varphi_{j}-\theta\right)\right)^{2}}{\left(1-2 r_{j} \cos \left(\varphi_{j}-\theta\right)+r_{j}^{2}\right)\left(1-2 r_{j}^{-1} \cos \left(\varphi_{j}-\theta\right)+r_{j}^{-2}\right)} \\
& +\sum_{j=k+1}^{u} \frac{1-r_{j} \cos \left(\varphi_{j}-\theta\right)}{1-2 r_{j} \cos \left(\varphi_{j}-\theta\right)+r_{j}^{2}} \\
& >0
\end{aligned}
$$


Proof of Theorem 2.12. As

$$
U_{n}^{\prime}\left(\tau_{k}\right)=(-1)^{k} B\left(n, \omega, \tau_{k}\right), \quad k=1,2, \ldots, 2 n,
$$

the main assumptions of the theorem may be read as

$$
\left|Q\left(\tau_{k}\right)\right| \leq\left|U_{n}^{\prime}\left(\tau_{k}\right)\right|, \quad k=1,2, \ldots, 2 n,
$$

and

$$
|Q( \pm \omega)| \leq\left|U_{n}^{\prime}( \pm \omega)\right|
$$

Suppose to the contrary that $|Q(\tau)|>\left|U_{n}^{\prime}(\tau)\right|$ for some $\tau \in\left[-\omega, \tau_{1}\right] \cup\left[\tau_{2 n}, \omega\right]$. Replacing $Q(\cdot)$ with $-Q( \pm \cdot)$, if necessary, without loss of generality we may assume that $\tau \in\left[\tau_{2 n}, \omega\right]$ and $Q(\tau)>U_{n}^{\prime}(\tau)$. But then $0 \neq R:=Q-(1+\varepsilon) U_{n}^{\prime}$ with a sufficiently small $\varepsilon>0$ has at least one zero on each of the intervals $\left(\tau_{1}, \tau_{2}\right),\left(\tau_{2}, \tau_{3}\right), \ldots,\left(\tau_{2 n-1}, \tau_{2 n}\right),\left(\tau_{2 n}, \tau\right)$, and $\left(\tau, \tau_{1}+2 \pi\right)$, and hence at least $2 n+1$ zeros in the period $[-\pi, \pi)$, a contradiction. Thus the conclusion of the theorem holds.

Proof of Lemma 2.13. As it is observed in [23] or on page 244 of [2] see Step 1), the assumption

$$
2 n>\left(3 \tan ^{2}(\omega / 2)+1\right)^{1 / 2}
$$

implies that $U^{\prime \prime}(\tau)>0$ for $\tau \in\left[\tau_{2 n}, \omega\right]$, and $U^{\prime \prime}(\tau)<0$ for $\tau \in\left[-\omega, \tau_{1}\right]$. Hence $U_{n}^{\prime}$ is increasing on $\left[\tau_{2 n}, \omega\right]$, and decreasing on $\left[-\omega, \tau_{1}\right]$. This, together with Theorem 2.12, yields that if $Q \in \mathcal{T}_{n}$ satisfies the assumptions of the theorem, then

$$
|Q(\tau)| \leq\left|U_{n}^{\prime}(\tau)\right| \leq\left|U_{n}^{\prime}( \pm \omega)\right|, \quad \tau \in\left[-\omega, \tau_{1}\right] \cup\left[\tau_{2 n}, \omega\right]
$$

On the other hand, since $B(n, \omega, \tau)$ is increasing on $[0, \omega)$ and decreasing on $(-\omega, 0]$, we have

$$
|Q(\tau)| \leq B(n, \omega, \tau) \leq B\left(n, \omega, \tau_{1}\right)=\left|U_{n}^{\prime}\left(\tau_{1}\right)\right|=\left|U_{n}^{\prime}\left(\tau_{2 n}\right)\right| \leq\left|U_{n}^{\prime}(\omega)\right|, \quad \tau \in\left[\tau_{1}, \tau_{2 n}\right]
$$

Combining the previous two inequalities with

$$
\left|U_{n}^{\prime}(\omega)\right|=2 n^{2} \cot (\omega / 2)
$$

gives the theorem.

\section{REFERENCES}

1. V.V. Andrievskii, Constructive function theory on sets of the complex plane through potential function theory and geometric function theory, Surveys in Approximation Theory 2 (2006), $1-52$.

2. P. Borwein and T. Erdélyi, Polynomials and Polynomial Inequalities, Springer, New York, 1995. 
3. P. Borwein and T. Erdélyi, A sharp Bernstein-type inequality for exponential sums, J. Reine Angew. Math. 476 (1996), 127-141.

4. T. Erdélyi, Markov-Bernstein type inequalities for polynomials under Erdős-type constraints, in "Paul Erdős and his mathematics I (Budapest, 1999)", Bolyai Soc. Math. Stud., 11, Gábor Halász, László Lovász, Dezső Miklós, and Vera T. Sós Eds., Springer Verlag, New York, 2002, pp. 219-239.

5. T. Erdélyi, Markov-Bernstein-type inequality for trigonometric polynomials with respect to doubling weights on $[-\omega, \omega]$, Constr. Approx. 19 (2003), no. 3, 329-338.

6. T. Erdélyi, Extremal properties of polynomials, in "A Panorama of Hungarian Mathematics in the XXth Century" János Horváth (Ed.), Springer, New York, 2005, pp. 119-156.

7. T. Erdélyi, Markov type estimates for derivatives of polynomials of special type, Acta Math. Hungar. 51 (3-4) (1988), 421-436.

8. T. Erdélyi, Markov and Bernstein type inequalities for certain classes of constrained trigonometric polynomials on an interval shorter than the period, Studia Sci. Math. Hungar. 25 (1990), 3-25.

9. T. Erdélyi and J. Szabados, On trigonometric polynomials with positive coefficients, Studia Sci. Math. Hungar. 24 (1989), 71-91.

10. T. Erdélyi and J. Szabados, Bernstein-type inequalities for a class of polynomials, Acta Math. Hungar. 52 (1989), 237-251.

11. C.K. Kobindarajah and D.S. Lubinsky, $L_{p}$ Markov-Bernstein inequalities on all arcs of the unit circle, J. Approx. Theory 116 (2002), no. 2, 343-368.

12. P. Lax, Proof of a conjecture of P. Erdös on the derivative of a polynomial, Bull. Amer. Math. Soc. 50 (1944), 509-513.

13. Xin Li, R.N. Mohapatra, and R.Z. Rodriguez, Bernstein-type inequalities for rational functions with prescribed poles, J. London Math. Soc. (2) 51 (1995), 523-531.

14. D.S. Lubinsky, $L_{p}$ Markov-Bernstein inequalities on arcs of the unit circle, J. Approx. Theory 108 (2001), no. 1, 1-17.

15. M.A. Malik, On the derivative of polynomials, J. London Math. Soc. 8 (1969), 57-60.

16. G.V. Milovanovic, D.S. Mitrinovic, and Th.M. Rassias, Topics in Polynomials: Extremal Problems, Inequalities, Zeros, World Scientific Publishing Co., Inc., River Edge, NJ, 1994..

17. B. Nagy and V. Totik, Sharpening of Hilbert's lemniscate theorem., J. Anal. Math. 96 (2005), 191223.

18. B. Nagy and V. Totik, Bernstein's inequality for algebraic polynomials on circular arcs, Constr. Approx. (to appear).

19. Q.I. Rahman and G. Schmeisser, Analytic theory of polynomials, London Mathematical Society Monographs. New Series, 26. The Clarendon Press, Oxford University Press, Oxford.

20. V. Totik, Polynomial inverse images and polynomial inequalities, Acta Math. 187 (2001), 139160.

21. V. Totik, Bernstein-type inequalities, J. Approx. Theory 164, no. 10, 1390-1401.

22. V. Totik, persponal communication.

23. V.S. Videnskii, Extremal estimates for the derivative of a trigonometric polynomial on an interval shorter than its period, Soviet Math. Dokl. 1 (1960), 5-8. 
24. V.S. Videnskii, On trigonometric polynomials of half-integer order, Izv. Akad. Nauk Armjan. SSR Ser. Fiz.-Mat. Nauk 17 (1964), 133-140.

Department of Mathematics, Texas A\&M University, College Station, Texas 77843

E-mail address: terdelyi@math.tamu.edu 